\title{
artigo
}

Araújo, H.V.S.; Lopes da Silva, R.; Gomes da Silva, I.M.; Santos, S.T.L.; Oliveira, C.D.B.; Bezerra, S.M.M.S.;

Assistência de enfermagem a mulheres acometidas por eclampsia e pré-eclampsia: revisão integrativa

\section{Assistência de enfermagem a mulheres acometidas por eclampsia e pré-eclampsia: revisão integrativa}

\author{
Nursing assistance to women affected by eclampsia and pre-eclampsia: revision integrativa \\ Asistencia de enfermería a mujeres afectadas por eclampsia y pre-eclampsia: revisión integrativa
}

\begin{abstract}
RESUMO
Objetivo: Buscar na literatura científica o que as evidências apontam sobre a assistência de enfermagem a mulheres acometidas por pré-eclampsia/eclampsia. Método: Trata-se de estudo de revisão de literatura integrativa conduzida nas bases de dados, LILACS, SciELO, MedLine e BDENF no período de outubro a novembro de 2020. Foram utilizados os seguintes descritores e sequência de busca: pré-eclampsia OR eclampsia AND cuidados de enfermagem. 0 corpus da análise compreendeu 5 artigos. Resultados: De acordo com os artigos selecionados e revisados, o rastreio precoce da pré-eclampsia/eclampsia é muito importante para minimizar os riscos de agravos, por isso o controle e acompanhamento da pressão arterial em gestantes são condutas indispensáveis. Conclusão: 0 estudo possibilitou analisar que a enfermagem é peça indispensável e de suma importância na equipe multidisciplinar, seja na unidade básica de saúde fazendo rastreio e diagnósticos de enfermagem precoces, ou em unidades de suporte avançado.
\end{abstract}

DESCRITORES: Cuidados de Enfermagem; Eclampsia; Pré-eclampsia.

\section{ABSTRACT}

Objective: To search the scientific literature for what the evidence points to nursing care for women affected by pre-eclampsia / eclampsia. Method: This is an integrative literature review study conducted in the databases, LILACS, SciELO, MedLine and BDENF from October to November 2020. The following descriptors and search sequence were used: pre-eclampsia OR eclampsia AND nursing care. The analysis corpus comprised 5 articles. Results: According to the selected and revised articles, the early screening for pre-eclampsia / eclampsia is very important to minimize the risk of health problems, so the control and monitoring of blood pressure in pregnant women are indispensable approaches. Conclusion: The study made it possible to analyze that nursing is an indispensable and extremely important part of the multidisciplinary team, whether in the basic health unit doing screening and early nursing diagnoses, or in advanced support units.

DESCRIPTORS: Nursing Care; Eclampsia; Pre-eclampsia.

\section{RESUMEN}

Objetivo: Buscar en la literatura científica lo que indica la evidencia sobre la atención de enfermería a mujeres afectadas por preeclampsia / eclampsia. Método: Se trata de un estudio de revisión integrativa de la literatura realizado en las bases de datos LILACS, SciELO, MedLine y BDENF de octubre a noviembre de 2020. Se utilizaron los siguientes descriptores y secuencia de búsqueda: preeclampsia 0 eclampsia Y cuidados de enfermería. El corpus de análisis estuvo compuesto por 5 artículos. Resultados: De acuerdo con los artículos seleccionados y revisados, el cribado precoz de preeclampsia / eclampsia es muy importante para minimizar el riesgo de problemas de salud, por lo que el control y seguimiento de la presión arterial en mujeres embarazadas son enfoques indispensables. Conclusión: El estudio permitió analizar que la enfermería es una parte indispensable y sumamente importante del equipo multidisciplinario, ya sea en la unidad básica de salud que realiza el cribado y diagnóstico temprano de enfermería, como en las unidades de apoyo avanzado.

DESCRIPTORES: Atención de enfermeira; Eclampsia; Pre-eclampsia.

RECEBIDO EM: 08/02/2021 APROVADO EM: 05/04/2021

\section{Hirla Vanessa Soares Araújo}

Enfermeira. Mestre em Enfermagem pelo Programa Associado de Pós Graduação em Enfermagem UPE/UEPB.

ORCID: 0000-0002-9995-7288 


\section{Rayane Lopes da Silva}

Acadêmica do curso de Bacharelado em Enfermagem do Centro Universitário dos Guararapes. ORCID: 0000-0002-0762-6909

\section{Isabelle Maria Gomes da Silva}

Acadêmica do curso de Bacharelado em Enfermagem do Centro Universitário dos Guararapes. ORCID: 0000-0003-2324-2005

\section{Samara Thais de Lima Santos}

Acadêmico do curso de Bacharelado em Enfermagem do Centro Universitário dos Guararapes. ORCID: 0000-0001-9942-488X

\section{Carmen Daniella Batista de Oliveira}

Enfermeira. Docente do curso de Bacharelado em Enfermagem do Centro Universitário dos Guararapes. ORCID: 0000-0002-7545-9241

\section{Simone Maria Muniz da Silva Bezerra}

Enfermeira. Pós-Doutora. Docente do Programa Associado de Pós Graduação em Enfermagem UPE/UEPB. ORCID: 0000-0002-0974-1409

\section{INTRODUÇÃO}

A gestação é um processo fisiológico em que modificações maternas acontecem de maneira temporária com a finalidade de nutrir e oxigenar o feto de acordo com as suas necessidades de desenvolvimento, por meio da ligação útero-placenta. No entanto, é importante saber fazer distinção entre o processo normal do período gravídico e o processo patológico ${ }^{1}$.

Em uma gravidez saudável ocorre um aumento no fluxo sanguíneo uteroplacentário, o que resulta em uma baixa resistência vascular sistêmica e diminuição da pressão arterial. Em complicações, como a pré-eclampsia, ocorre o contrário, ou seja, o fluxo sanguíneo uteroplacentário se encontra em níveis baixos levando a ocorrência de aumento na resistência vascular sistêmica e, consequentemente, da pressão arterial, o que é considerado um processo patológico alarmante ${ }^{1}$.

Segundo a Sociedade Brasileira de Cardiologia, a hipertensão arterial sistêmica (HAS) é considerada um problema de saúde pública mundial. A HAS é caracterizada pelo aumento elevado e sustentado da pressão arterial maior ou igual que 140/90mmHg. Na maioria dos casos, está relacionada com alterações estruturais e/ ou funcionais a ponto de atingir órgãos como o coração, encéfalo, rins e vasos san- guíneos. Essas alterações também podem ter caráter metabólico, como por exemplo os eventos cardiovasculares ${ }^{2}$.

As Síndromes Hipertensivas na Gestação (SHG) representam a terceira causa no mundo e primeira no Brasil de morte materna. Essas síndromes geralmente evoluem sem dar sinais de intercorrências. No entanto, em outra parcela de gestantes podem se caracterizar por modificações que podem colocar a vida da gestante e do feto em risco ${ }^{3}$.

No período da gravidez é possível desenvolver algumas patologias que estão diretamente ligadas ao período gravídico, como Hipertensão induzida pela gravidez, Pré-eclampsia e Eclampsia, hipertensão crônica e hipertensão com toxemia. Essas patologias são causadoras de retardo do crescimento intra-uterino, prematuridade, descolamento prematuro da placenta, morte materno-fetal, dentre outras complicações ${ }^{3}$.

Dados da Organização Mundial da Saúde apontam que um quarto das mortes maternas na América Latina está associado a esses distúrbios. Entre eles, a pré-eclampsia e a eclampsia têm o maior impacto na morbidade e mortalidade materno-infantil ${ }^{4}$. No Brasil, cerca de $3 \%$ a $7 \%$ das gestantes apresentam quadro de pré-eclampsia 5 .

A pré-eclampsia acomete as gestantes após a vigésima semana da gestação e é caracterizada pelo desenvolvimento de hipertensão gradual (igual ou maior que 140/90 $\mathrm{mmHg}$ ) e proteinúria (maior que $300 \mathrm{mg} / 24 \mathrm{~h}$ ) em mulheres que nunca apresentaram aumento da pressão arterial. Sua classificação se dá de acordo com os sintomas apresentados. Quando se identifica um aumento elevado de peso é classificada como leve. Já a grave ocorre quando apresenta um ou mais dos sintomas anteriormente citados, oligúria menor que $500 \mathrm{ml} / \mathrm{dia}$, ou $25 \mathrm{ml} /$ hora, plaquetopenia $<100.000 / \mathrm{mm}$ e dor epigástrica ou no hipocôndrio direito ${ }^{6}$.

Quando há existência de convulsões na mulher com pré-eclampsia, o processo denomina-se eclampsia. Além disso, soma-se sinais como enxaqueca, cefaleia occipital e dor abdominal intensa, caracterizando-a como uma emergência obstétrica ${ }^{7}$.

No que diz respeito aos fatores de risco, destacam-se o aumento de peso, idade materna menor que 15 e maior que 35 anos, edema, histórico familiar, nuliparidade. Em relação ao aumento de peso, este possui um grande impacto na saúde da gestante e maior é o risco para o desenvolvimento de pré-eclampsia. A faixa etária materna (menor que 15 anos/ maior que 35 anos) e a presença de edema, principalmente quando localizado nos membros inferiores, também são sinais de alerta. Uma mulher nulípara apresentam riscos maiores comparados a uma mulher 
que já tem filhos. Histórico familiar de pessoas com parentesco de primeiro grau, como mãe ou irmã, que tiveram relatos de pré-eclampsia também se configura como fator de risco ${ }^{3}$.

A gestante diagnosticada com pré-eclampsia pode apresentar muitas complicações que são caracterizadas pelo acometimento de sistemas vitais como: alterações hepáticas, cerebrais, sanguíneas, hidroeletrolíticas e uteroplacentárias. O quadro pode evoluir para eclampsia, que eleva o risco em relação à mortalidade. Cabe ressaltar que essas complicações podem resultar em sequelas, acometendo o feto ou ambos. Por essa razão exige uma maior atenção na assistência à gestante ${ }^{8}$.

Já os distúrbios causados pela eclampsia podem ocorrer no parto e até 10 dias no puerpério. Geralmente, as mortes maternas por eclampsia ocorrem por complicações como: acidentes vasculares cerebrais, insuficiência cardíaca congestiva ou complicações cirúrgicas ${ }^{3}$.

É necessário realizar avaliação fetal atentando para os danos causados pela pré-eclampsia e eclampsia e avaliar se o feto tem desenvolvimento e crescimento normais, bem como solicitar exame ultrassonográfico em um intervalo de 2 a 4 semana para reavaliação ${ }^{6}$.

Destaca-se a atuação da enfermagem frente à mulher acometida por síndrome hipertensiva durante o período gravídico-puerperal. $\mathrm{O}$ enfermeiro além de seguir os protocolos também deve acolher a gestante nas emergências, de maneira holística. A revisão do histórico gestacional é indispensável para a identificação correta de patologias no período gravídico para posterior elaboração do plano de cuidados. $\mathrm{O}$ profissional deve se atentar que pacientes diagnosticadas com a pré-eclampsia não podem fazer tratamento com tocolíticos, que é a supressão farmacológica do parto prematuro antes da trigésima sétima semana de gravidez?

A abordagem gerada de acordo com métodos baseados em evidências científicas é indispensável e proporciona maior qualidade no acolheimento e aprimoramento dos resultados positivos de forma a garantir a segurança e confiabilidade do atendimento ${ }^{10}$.

Diante desse contexto, o objetivo da pesquisa foi identificar na literatura o que as evidências científicas apontam sobre a assistência de enfermagem a mulheres acometidas por pré-eclampsia/eclampsia.

\section{MÉTODO}

A metodologia utilizada para a pesquisa foi uma revisão integrativa da literatura, que tem como alvo sintetizar informações baseando-se nas técnicas de coleta de dados de pesquisas bibliográficas, permitindo uma análise mais profunda da assistência a mulheres acometidas por pré-eclampsia/eclampsia, contribuindo para a prática de enfermagem baseada em evidências científicas. Este método possibilitou analisar e compreender de forma abrangente o que há na literatura sobre o tema a partir da seguinte questão norteadora: $\mathrm{O}$ que as evidências científicas apontam sobre a assistência de enfermagem a mulheres acometidas por pré-eclampsia/ eclampsia?

A partir desse questionamento, procedeu-se às demais etapas para realização da pesquisa: formulação dos critérios de inclusão, definição das informações a serem extraídas das pesquisas selecionadas, avaliação rigorosa dos estudos incluídos na revisão integrativa, interpretação dos resultados e síntese do conhecimento ${ }^{11}$.

As orientações foram seguidas de acordo com o Preferred Reporting Items for Systematic Reviews and Meta-Analyses (PRISMA) ${ }^{12}$.

Para a seleção dos estudos, foram pesquisadas as bases de dados: Medical Literature Analysis and Retrieval System Online (MEDLINE), Literatura Latino Americana e do Caribe em Ciências da Saúde (LILACS), Scientific Eletronic Library Online (SciELO) e Banco de Dados em Enfermagem (BDENF), no período de outubro a novembro de 2020. As bases de dados foram escolhidas pela sua grande abrangência de estudos. Realizou-se a seleção de descritores após consulta ao DeCS/ $\mathrm{MeSH}$. Os descritores utilizados e suas combinações foram: pré-eclampsia OR eclampsia AND cuidados de enfermagem.

Obedeceu-se a mesma sequência na inserção dos descritores para as buscas nas bases de dados, delimitando-se em cinco anos o período dos estudos, com o objetivo de abranger o maior quantitativo de publicações a respeito da temática. Os artigos selecionados obedeceram aos seguintes critérios de inclusão: pesquisas disponíveis eletronicamente nas bases de dados selecionados, publicadas em periódicos revisados por pares nos últimos cinco anos, textos completos disponíveis na íntegra e gratuitamente nos idiomas português, inglês e espanhol e publicados nos últimos cinco anos. Foram excluídos artigos, matérias de revistas e livros completos que não respondessem à questão norteadora do estudo.

A leitura seletiva dos artigos foi realizada, inicialmente, com análise do título e resumo. Os artigos duplicados foram registrados apenas uma vez. Para garantir o registro conjunto de informações relevantes ao tema foi utilizado instrumento próprio para análise dos artigos, o qual incluía dados de identificação (período publicado, formação e instituição à qual os autores estão vinculados, idioma e país), aspectos metodológicos dos estudos (delineamento da pesquisa, amostragem e tratamento dos dados) e principais resultados e conclusões. Em seguida, realizou-se análise detalhada dos artigos, considerando o rigor e as características dos mesmos, seguindo-se ao registro das informaçốes elencadas no instrumento. As informações foram interpretadas, resumidas e organizadas em quadro síntese, comparando-se os resultados, e finalizadas com proposições acerca dos estudos.

Inicialmente foram encontrados 51 artigos, sendo 2 excluídos por duplicata. Após a leitura dos títulos e resumos foram excluídas 20 pesquisas. Após a leitura na íntegra dos estudos, 13 foram excluídos. O corpus da análise foi de 5 artigos.

Os artigos selecionados foram submetidos à classificação do nível de evidência constituída por sete níveis: I) evidências obtidas de revisão sistemática ou meta-a- 
nálise de ensaios clínicos randomizados, controlados ou diretrizes clínicas baseadas em revisões sistemáticas de ensaios clínicos randomizados controlados; II) evidências oriundas de pelo menos um ensaio clínico randomizado, controlado, bem delineado; III) evidências provenientes de ensaios clínicos, sem randomização, bem delineados; IV) evidên- cias obtidas de estudos de coorte e caso controle, bem delineados; V) evidências que tiveram origem de revisão sistemática de estudos descritivos e qualitativos; VI) evidências oriundas de um único estudo descritivo ou qualitativo e; VII) evidências provenientes de opinião de autoridades ou relatório de comitês de especialistas. Segundo esta classificação, os níveis 1

Figura 1 - Fluxograma da seleção dos artigos e motivos de exclusão, Jaboatão dos Guararapes/PE. 2020

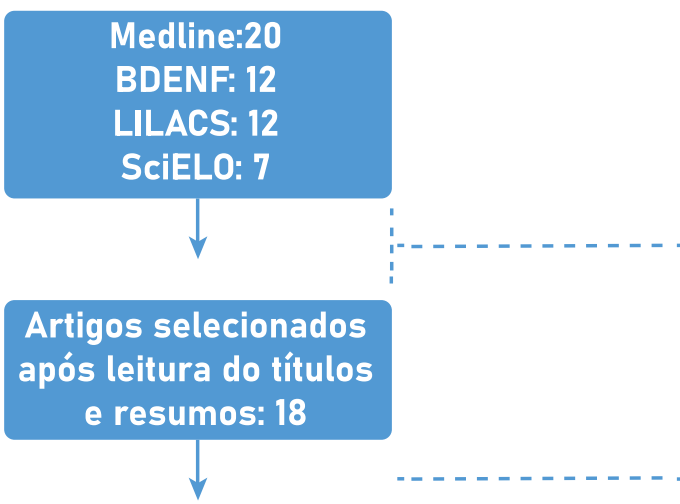

Artigos selecionados

para a pesquisa: 6

e 2 são considerados evidências fortes, 3 e 4 moderadas e 5 a 7 fracas $^{13}$.

\section{RESULTADOS}

Foram encontrados 20 artigos na Medline, 12 na BDENF, 12 no LILACS e 7 no SciELO. A figura 1 apresenta o fluxograma da estratégia de seleção dos artigos conforme os critérios de elegibilidade pré-estabelecidos.

No quadro 1 é apresentada a caracterização da análise dos artigos referentes a título, país, ano de publicação, desenho do estudo, amostra e resultados principais. Os artigos foram publicados entre os anos de 2016 e 2020, sendo 3 artigos de revisão integrativa (níveis de evidência $\mathrm{V}$ ),3 estudos descritivos (nível de evidência VI). A maioria dos estudos (5) foram desenvolvidos no Brasil e 1 na Itália.

\section{DISCUSSÃO}

Para melhor apresentação da discussão a respeito dos resultados desta revisão integrativa, optou-se pela sua divisão em duas categorias: Perfil de gestantes aco-

Quadro 1 - características dos estudos selecionados, Jaboatão dos Guararapes/PE. 2020

\begin{tabular}{|c|c|c|c|c|}
\hline TÍTULO & $\begin{array}{l}\text { PAÍS/ } \\
\text { ANO }\end{array}$ & $\begin{array}{c}\text { DESENHO DO ESTUDO E } \\
\text { AMOSTRA }\end{array}$ & RESULTADOS PRINCIPAIS & $\begin{array}{l}\text { NIVELL DE } \\
\text { EVIDÊNCIA }\end{array}$ \\
\hline $\begin{array}{l}\text { Influence of Estrogens } \\
\text { on Uterine Vascular } \\
\text { Adaptation in Normal } \\
\text { and Preeclamptic Preg- } \\
\text { nancies }{ }^{1} \text {. }\end{array}$ & $\begin{array}{l}\text { Itália, } \\
2020\end{array}$ & $\begin{array}{l}\text { Revisão integrativa. Amostra } \\
\text { de } 157 \text { artigos }\end{array}$ & $\begin{array}{c}\text { Observou-se que os níveis mais baixos de estrogênio } \\
\text { no plasma e a regulação negativa de REs que têm } \\
\text { sido frequentemente observados em gestantes com } \\
\text { pré-eclampsia. }\end{array}$ & V \\
\hline $\begin{array}{l}\text { Perfil de gestantes com } \\
\text { síndrome hipertensiva } \\
\text { em uma maternidade } \\
\text { pública }{ }^{3} \text {. }\end{array}$ & $\begin{array}{l}\text { Brasil, } \\
2016\end{array}$ & $\begin{array}{l}\text { Estudo descritivo, } 468 \\
\text { prontuários de gestantes } \\
\text { submetidas à cesariana no } \\
\text { período de janeiro de } 2008 \\
\text { a dezembro de } 2012 \text {. }\end{array}$ & $\begin{array}{c}\text { Observou-se que as síndromes hipertensivas } \\
\text { gestacional merecem especial destaque na saúde } \\
\text { pública iniciando com pré-natal }\end{array}$ & VI \\
\hline $\begin{array}{l}\text { Assistência de enfer- } \\
\text { meiros na síndrome } \\
\text { hipertensiva gestacional } \\
\text { em Hospital de baixo } \\
\text { risco obstétrico }{ }^{8} \text {. }\end{array}$ & $\begin{array}{l}\text { Brasil, } \\
2017 .\end{array}$ & $\begin{array}{c}\text { Pesquisa de campo, amostra } \\
\text { de } 9 \text { enfermeiros. }\end{array}$ & $\begin{array}{c}\text { Evidenciou a análise da assistência de enfermeiros } \\
\text { às gestantes com síndrome hipertensiva, sendo essa } \\
\text { atenção essencial na preservação e manutenção da } \\
\text { vida da mulher e do feto. }\end{array}$ & VI \\
\hline 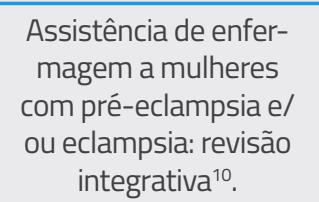 & $\begin{array}{l}\text { Brasil, } \\
2016\end{array}$ & $\begin{array}{l}\text { Revisão integrativa, amostra } \\
\text { de } 17 \text { artigos primários. }\end{array}$ & $\begin{array}{c}\text { O estudo possibilitou caracterizar o perfil das gestan- } \\
\text { tes acometidas de pré-eclampsia internadas em uma } \\
\text { maternidade pública. }\end{array}$ & V \\
\hline
\end{tabular}




\begin{tabular}{|c|c|c|c|c|}
\hline $\begin{array}{c}\text { Perfil de gestantes com } \\
\text { pré-eclampsia }^{14}\end{array}$ & $\begin{array}{c}\text { Brasil, } \\
\text { 2017. }\end{array}$ & $\begin{array}{c}\text { Estudo descritivo, amostra } \\
\text { de } 28.399 \text { mulheres que de- } \\
\text { ram entrada na maternidade } \\
\text { nos anos de 2013 e 2014 }\end{array}$ & $\begin{array}{c}\text { Evidenciou a importância dos cuidados de enferma- } \\
\text { gem específicos a mulheres com pré-eclampsia e } \\
\text { eclampsia quais são capazes de reduzir complicações e } \\
\text { taxas de morbimortalidade. }\end{array}$ & $\begin{array}{c}\text { VI } \\
\text { Pré-eclampsia/eclamp- } \\
\text { sia }^{15}\end{array}$ \\
\hline $\begin{array}{c}\text { Brasil, } \\
2020\end{array}$ & $\begin{array}{c}\text { Revisão sistemática, amos- } \\
\text { tra de } 60 \text { artigos }\end{array}$ & $\begin{array}{c}\text { Dados disponibilizados entre 2002 e 2010 demons- } \\
\text { trou incidência variando de 1,2\% a 4,2\% para pré-e- } \\
\text { clampsia e de 0,1\% a 2,7\% para eclampsia, e as taxas } \\
\text { mais elevadas foram identificadas em regiões de } \\
\text { menor desenvolvimento socioeconômico. }\end{array}$ \\
\hline Fonte: dados da pesquisa, 2020.
\end{tabular}

metidas pela pré-eclampsia/eclampsia e Cuidados de enfermagem à gestante com pré-eclampsia e eclampsia.

\section{Categoria 1: Perfil de gestantes acometidas pela pré-eclampsia/ eclamspia}

Em relação ao perfil das gestantes acometidas por pré-eclampsia e eclampsia, a faixa etária predominante é entre 15 e 25 anos. A maioria é solteira, de baixa escolaridade e não apresentava ocupação profissional relatada. No tocante à idade, um dos fatores de alerta para complicações durante a gravidez são os extremos de idade, pois quando se é jovem demais ou quando se tem uma idade mais avançada há o risco de desenvolver pré-eclampsia ou eclampsia ${ }^{15}$.

Em relação à baixa escolaridade e à baixa renda, condiçôes socioeconômicas desfavoráveis têm levado as mulheres à uma gestação de alto risco, uma vez que a situação pode provocar estresse e piores condições nutricionais. Atrelada à condição nutricional, um estudo que avaliou o perfil antropométrico de gestantes internadas com diagnóstico de pré-eclampsia grave indica que a inadequação da nutrição materna durante o período da gestação constitui um problema de saúde pública, uma vez que é fator de risco que pode favorecer o aparecimento de intercorrências durante a gravidez, a exemplo da diabetes e pré-eclampsia ${ }^{14,15}$.

Estudo sobre atenção ao pré-natal e fatores de risco associados à prematuridade e baixo peso ao nascer corrobora ao afirmar que a baixa escolaridade entre mães corresponde a um agravante para a saúde das mulheres, sendo considerado um fator de risco obstétrico ${ }^{16}$.

\section{Estudo sobre}

atenção ao

pré-natal e fatores

de risco associados

à prematuridade $\mathrm{e}$

baixo peso ao

nascer corrobora

ao afirmar que a

baixa escolaridade

entre mães

corresponde a um

agravante para a

saúde das mulheres,

sendo considerado

um fator de risco

obstétrico
Quanto ao estado civil, corroborando com os achados desta revisão integrativa, a literatura relata que há predominância da síndrome hipertensiva gestacional em jovens entre 15 e 19 anos, idade essa em que muitas se encontram solteiras ${ }^{3}$.

Em relação ao perfil clínico, dentre os antecedentes familiares, a incidência da hipertensão é muito frequente, seguida de Diabetes mellitus, gemelaridade e pré-eclampsia ${ }^{14}$. Segundo estudo sobre características maternas e fatores de risco para pré-eclampsia em gestantes, a anamnese e exame físico são excelentes preditores obstétricos, corroborando, desta forma, para a história pessoal da gestante em busca dessas informações ${ }^{17}$.

Categoria 2: Cuidados de enfermagem à gestante com pré-eclampsia/ eclampsia

A assistência de enfermagem em gestantes com pré-eclampsia e eclampsia é de grande importância para o rastreio precoce. A primeira técnica que deve ser padronizada é a aferição da pressão arterial durante a consulta de enfermagem para verificar um dos primeiros sintomas apresentados por esta patologia. Durante o seguimento da investigação, quando há elevação dos níveis pressóricos, são solicitados outros exames complementares para confirmar o diagnóstico. A aferição da pressão arterial é importante para um prognóstico correto, devendo ser realizada de forma adequada para evitar falso negativo ou falso positivo. Sua importância se dá por ser forte indicador para diagnóstico de Síndrome Hipertensiva Gestacional, tronando-se indispensável para controle e acompanhamento da pressão arterial em gestantes ${ }^{10}$. 
O enfermeiro durante a consulta de pré-natal na Unidade Básica de Saúde deve realizar a anamnese e exame físico em todas as gestantes, pesquisando por sintomas e queixas que elas possam apresentar durante o período gestacional e, caso apresente fatores de risco, deve ser destacado no Cartão da Gestante para alertar outros profissionais de saúde que venham a fazer seu acompanhamento. Ao levantar a suspeita de pré-eclampsia grave durante a consulta do pré-natal, o enfermeiro deve encaminhar a gestante para um pré-natal de alto risco ou unidade de referência hospitalar obstétrica, devendo observar também se a gestante é asmática com sintomas de moderados a graves, pois há um risco maior de apresentar sintomas do agravo em questão ${ }^{18}$.

Durante as consultas no pré-natal de baixo risco o enfermeiro deve estar sempre atento ao avaliar o aumento progressivo de peso da gestante e verificar a existência de edema, especialmente após a $24^{\circ}$ semana, pois gestante com ganho de peso igual ou maior que $500 \mathrm{~g}$ por semana, mesmo sem elevação da pressão arterial, deve ter acompanhamento contínuo. A presença de edema ocorre em $80 \%$ das gestantes com prognóstico para pré-eclampsia, onde a conduta do enfermeiro deve ser de acompanhar e orientar repouso em decúbito dorsal esquerdo e avaliar movimentos fetais ${ }^{5}$.

Os enfermeiros que atuam na emergência/urgência obstétrica avaliam e estabilizam a gestante por meio da classificação de risco. A partir de então é realizado o devido encaminhamento que, para tanto, é preciso que a equipe realize um exame físico minucioso, conhecendo o histórico da paciente para melhor avaliação e detecção precoce. Desta forma, caso a equipe identifique qualquer situação de risco ela irá intervir rapidamente. Ainda, se durante a avaliação da gestante a mesma apresenta alteração na pressão arterial, deverá ser solicitado exame de urina para verificação da presença ou não de proteinúria ${ }^{18}$.

Quando diagnosticada com pré-eclampsia leve, a gestante é hospitalizada e mantida em observação. Uma dieta nor- mossódica pode ser adotada pela nutricionista. Já em casos de pré-eclampsia grave, a conduta dependerá da gravidade e da idade gestacional da paciente. A administração de sulfato de magnésio é realizada pela equipe de enfermagem na pré-eclampsia grave e eclampsia, conforme prescrição médica, de acordo com o valor da pressão arterial, devendo o profissional ficar atento a alergias ${ }^{18}$.

Outros cuidados de enfermagem envolvem a monitorização e observação da

\section{Os enfermeiros}

que atuam na

emergência/urgência

obstétrica avaliam

e estabilizam a

gestante por meio da

classificação de risco.

A partir de então é

realizado o devido

encaminhamento

que, para tanto, é

preciso que a equipe

realize um exame

físico minucioso,

conhecendo o

histórico da paciente

para melhor

avaliação e

detecção precoce. paciente quando ela se encontra em indução do trabalho de parto, além de explicar a importância de medidas adotadas. Essas condutas ajudam a minimizar os níveis de ansiedade e o medo da gestante. Se a paciente evolui para a gravidade, estudos evidenciam cuidados como: mudança de decúbito para lateral esquerdo, oxigenioterapia, acesso venoso, administração dos medicamentos conforme prescrição médica, prevenção de infecções e agravos, preparo e administração correta do sulfato de Magnésio, instalação de uma Cânula de Guedel (dispositivo que mantém pérvia a via aérea em pacientes inconscientes ou com algum rebaixamento do nível de consciência) ${ }^{10}$.

Deve-se lembrar de que prestar uma assistência à gestante é necessário incluir a avaliação fetal. Para tanto, pode ser utilizado o partograma. Trata-se de documento que faz parte do prontuário e que deve ser preenchido a partir do momento que a paciente entra em trabalho de parto com todas as informações necessárias, incluindo frequência cardíaca do feto. A monitoração do bem-estar fetal e da puérpera deve ser rigorosa e constante por no mínimo 24 a 48 horas, podendo esse período se estender a depender da evolução do quadro. $\mathrm{O}$ uso de sulfato de magnésio deve ser continuado até 24 horas após o parto, avaliando estabilização da pressão arterial. Em casos de níveis pressóricos elevados, a terapia anti-hipertensiva é mantida ${ }^{13}$.

Não obstante, alguns pontos se mostraram relevantes por corroborar com uma assistência de baixa qualidade à gestante como: início tardio do atendimento pré-natal, não verificação da pressão arterial no primeiro trimestre, suporte inadequado para atender a paciente, falta de infraestrutura, falta de informação que deve ser passada de forma clara, falta de treinamento e habilidade dos profissionais ${ }^{10}$.

Ao realizar essa pesquisa observou-se a importância do conhecimento que irá impulsionar a equipe multidisciplinar a oferecer um serviço de qualidade para essas gestantes, onde o objetivo do enfermeiro, ao fazer o acompanhamento da gestante acometida pelo agravo em questão, é redu- 
zir os riscos materno/fetal. Uma portaria que pode guiar este profissional durante sua assistência no âmbito do Sistema Único de Saúde é a Portaria de número 1.459/ GM/MS/2011, criada pelo Ministério da Saúde e denominada Rede Cegonha, que tem como objetivos a redução da mortalidade materna e neonatal, acolhimento de intercorrências na gravidez, classificação de risco e acesso ao alto risco em tempo oportuno ${ }^{6}$.

Dentro desse contexto, percebe-se que o enfermeiro ao lidar com gestantes acometidas com eclampsia/pré-eclampsia desenvolve inúmeras tarefas com alto grau de responsabilidade e complexidade, onde prestar uma assistência de forma correta irá contribuir para redução das complica-
A maioria dos

estudos traz que há

uma predominância

de jovens mulheres,

solteiras, de baixa e

escolaridade e sem

ocupação relatada

acometidas pela pré-

eclampsia/eclampsia. ções provenientes desta enfermidade. Ter conhecimento científico e técnico contribui para intervenções adequadas, humanizadas e efetivas 5 .

\section{CONCLUSÃO}

A maioria dos estudos traz que há uma predominância de jovens mulheres, solteiras, de baixa e escolaridade e sem ocupação relatada acometidas pela pré-eclampsia/ eclampsia. Conhecer o perfil da mulher durante o período gravídico-puerperal é imprescindível para utilização da sistematização da assistência de enfermagem baseada nas reais necessidades da gestante, conferindo maior segurança ao binômio gestante-feto.

\section{REFERÊNCIAS}

1. Mandalà M. Influence of Estrogens on Uterine Vascular Adaptation in Normal and Preeclamptic Pregnancies. Int J Mol Sci. 2020; 21(7): 2592. DOI: https://doi.org/10.3390/ijms21072592

2. BRASIL. Ministério da Saúde. Estratégias para o cuidado da pessoa com doença crônica: hipertensão arterial sistêmicaBrasília. Cadernos de Atenção Básica, n. 37, 2014.

3. Nóbrega MF, Santos MTBR, Davim RMB, Oliveira LFM, Alves ESRC, Rodrigues ESRC. Perfil de Gestantes com Síndrome Hipertensiva em uma Maternidade Pública. Rev enferm UFPE online. 2016; 10(5): 1805-11. DOI: 10.5205/reuol.9003-78704-1SM.1005201629.

4. ORGANIZAÇÃO MUNDIAL DA SAÚDE - Recomendações da OMS para a prevenção e tratamento da hemorragia pós-parto $-2014$.

5. BRASIL. Ministério da Saúde. Secretaria de Atenção à Saúde. Manual de acolhimento e classificação de risco em obstetríciaBrasília, 2017.

6. BRASIL. Ministério da Saúde. Secretaria de Atenção à Saúde. Gestação de Alto Risco: Manual Técnico. 5 ed. Brasília, 2010.

7. BRASIL. Ministério da Saúde- Protocolos da Atenção Básica. Instituto Sírio-Libanês de Ensino e Pesquisa - Brasília, 2016.

8. Oliveira GS, Paixão GP, Fraga CDS, Santos MKR, Santos MA. Assistência de enfermeiros na síndrome hipertensiva gestacional em hospital de baixo risco obstétrico. Rev Cuid. 2017; 8(2): 1561-72. DOI: http://dx.doi.org/10.15649/cuidarte.v8i2.374

9. Bulechek GM, Butcher HK, Dochterman JM. Classificações das Intervenções de Enfermagem (NIC). Tradução da $5^{\circ}$ Edição. Editora: Elsevier Editora Ltda - 2010.

10. Ferreira MBG, Silveira CF, Silva SR, Souza DJ, Ruiz MT. Nursing care for women with pre-eclampsia and/or eclampsia: integrative review. Ver Esc Enferm USP. 2016; 50(2): 320-330. DOI:
http://dx.doi.org/10.1590/S0080-623420160000200020.

11. Mendes KDS, Silveira RCCP, Galvão CM. Integrative literature review: a research method on corporate evidence in healthcare and nursing. Texto Contexto Enferm, 2008; 17(4): 758-64. DOI: 10.1590/S0104-07072008000400018.

12. Singh S. How to Conduct and Interpret Systematic Reviews and Meta-Analyses. Journal of the American College of Gastro-enterology. 2017 [14 jun 2020]; 8, e93. Disponivel em: <www.nature.com/ctg>.

13. Stillwell SB, Fineout-Overholt E, Melnyk BM, Williamson KM. Searching for the Evidence. Am J Nurs, 2010; 110(5): 41-7.

14. Amorim FCM, Neves ACN, Moreira FS, Oliveira ADS, Nery IS. Perfil de gestantes com pré-eclâmpsia. Ver enferm UFPE online. 2017; 11(4): 1574-83. DOI: 10.5205/reuol.9763-85423-1SM.1104201703

15. Barros Júnior FS, Barradas Júnior AR, Lima JC, Aires IO, Rocha CB, Rêgo Neta MM, Jacobina PKF. Perfil antropométrico de gestantes internas com diagnóstico de pré-eclâmpsia grave. 2019; 9(3):e199309. DOI: 10.15210/jonah.v9i3.16812.

16. Gonzaga ICA, Santos SLD, Silva ARV, Campelo V. Atenção pré-natal e fatores de risco associados à prematuridade e baixo peso ao nascer em capital do nordeste brasileiro. Ciênc Saúde Coletiva. 2016; 21(6). DOI: 10.1590/141381232015216.06162015.

17. Ferreira ETM, Moura NS, Gomes MLS, Silva EG, Guerreiro MGS, Oriá MOB. Características maternas e fatores de risco para pré-eclâmpsia em gestantes. Rev Rene. 2019; 20(1): e40327. DOI: 10.15253/2175-6783.20192040327.

18. BRASIL. Ministério da Saúde. Secretaria de Atenção à Saúde. Departamento de Atenção Básica. Atenção ao pré-natal de baixo risco - Brasília - Cadernos de Atenção Básica, n 32, 2012. 